\title{
Sub-wavelength metamaterial cylinders with multiple dipole resonances
}

\author{
Arslanagic, Samel; Breinbjerg, Olav
}

Published in:

IEEE International Symposium on Antennas \& Propagation \& USNC/URSI National Radio Science Meeting

Link to article, DOI:

10.1109/APS.2009.5172269

Publication date:

2009

Document Version

Publisher's PDF, also known as Version of record

Link back to DTU Orbit

Citation (APA):

Arslanagic, S., \& Breinbjerg, O. (2009). Sub-wavelength metamaterial cylinders with multiple dipole resonances. In IEEE International Symposium on Antennas \& Propagation \& USNC/URSI National Radio Science Meeting IEEE. https://doi.org/10.1109/APS.2009.5172269

\section{General rights}

Copyright and moral rights for the publications made accessible in the public portal are retained by the authors and/or other copyright owners and it is a condition of accessing publications that users recognise and abide by the legal requirements associated with these rights.

- Users may download and print one copy of any publication from the public portal for the purpose of private study or research

- You may not further distribute the material or use it for any profit-making activity or commercial gain

- You may freely distribute the URL identifying the publication in the public portal 


\title{
Sub-Wavelength Metamaterial Cylinders With Multiple Dipole Resonances
}

\author{
Samel Arslanagić* and Olav Breinbjerg, \\ Department of Electrical Engineering, Technical University of Denmark, \\ Building 348, Ørsteds Plads, DK-2800 Kgs. Lyngby, Denmark, \\ Tel: +45 4525 3800, Fax: +45 4593 1634; E-mail: sar@elektro.dtu.dk
}

\section{Introduction}

Certain classes of metamaterials (MTMs) such as double- and single-negative (DNG and SNG) materials, and their combinations with double-positive (DPS) materials, can offer sub-wavelength resonances in individual objects of various canonical shapes [1]-[4]. A collection of such MTM-based individual objects was studied in [1] with the aim of devising an effective hybrid MTM, in [5] for cloaking purposes, and in [6] for its scattering properties. For isolated cylindrical objects, it was shown in [2] that a set of concentric circular MTM cylinders excited by a near-by electric line current possesses sub-wavelength resonances where the excitation of specific modes leads to large radiated power for constant line current. This work investigates how these resonances of individual objects are affected when several objects are combined. To this end, configurations consisting of 2 and 4 resonant cylindrical objects are analyzed numerically and it is shown that these configurations recover the resonances of the isolated objects even when the objects are closely spaced and the configuration thus electrically small. The analysis includes the near-field distribution as well as the radiation resistance in the case of simple, but lossy and dispersive, MTMs. The time factor $\exp (j \omega t)$, with $\omega$ being angular frequency and $t$ time, is assumed and suppressed.

\section{Theory}

Figure 1 shows the $k^{\prime}$ th cylinder $(C k)$ of the 2- or 4cylinder configurations investigated in this work. A circular cylinder (region $i, C k$ ) with center at $O_{C k}$ and radius $\rho_{i, C k}$ is covered by a concentric circular shell (region $o, C k$ ) of outer radius $\rho_{o, C k}$, and located in free space with the permittivity $\varepsilon_{0}$ and the permeability $\mu_{0}$. Region $i, C k(o, C k)$ consists of simple, lossy, and dispersive DPS, DNG, or SNG materials with the permittivity $\varepsilon_{i, C k}=\varepsilon_{i, C k}^{\prime}-j \varepsilon_{i, C k}^{\prime \prime}\left(\varepsilon_{o, C k}=\varepsilon_{o, C k}^{\prime}-j \varepsilon_{o, C k}^{\prime \prime}\right)$ and the permeability $\mu_{i, C k}=\mu_{i, C k}^{\prime}-j \mu_{i, C k}^{\prime \prime} \quad\left(\mu_{o, C k}=\right.$ $\left.\mu_{o, C k}^{\prime}-j \mu_{o, C k}^{\prime \prime}\right)$.

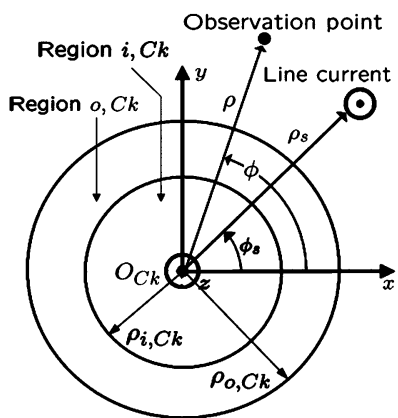

Figure 1. The $k^{\prime}$ th cylinder of the configuration.

The 1-, 2-, or 4-cylinder configuration is illuminated by an infinite electric line current (LC) $I_{e}$ that is parallel to the cylinders and can be located in any of the regions. The cylindrical $(\rho, \varphi, z)$-coordinate system and the Cartesian $(x, y, z)$-coordinate system are introduced with the $z$-axis coinciding with the common axis of the cylinders. The coordinates of the $\mathrm{LC}$ are $\left(\rho_{s}, \varphi_{s}\right)$.

The 1-cylinder configuration consists of a cylinder $C 1$ which has its center at the origin. i.e., $O_{C 1}=(0,0)$. The 2-cylinder configuration consists of the previous cylinder $C 1$ 
and a cylinder $C 2$ with center at $O_{C 2}=\left(\rho_{o, C 1}+d+\rho_{o, C 2}, 0\right)$, i.e., the cylinder $C 2$ is placed along the $x$-axis with a separation distance $d$ to the cylinder $C 1$. The 4-cylinder configuration consists of the previous cylinders $C 1$ and $C 2$, and the additional cylinders $C 3$ and $C 4$ having the respective centers at $O_{C 3}=\left(\rho_{o, C 1}+d / 2, d / 2+\rho_{o, C 3}\right)$ and $O_{C 4}=$ $\left(\rho_{o, C 1}+d / 2,-d / 2-\rho_{o, C 4}\right)$.

For all configurations, a numerical solution has been obtained using the HFSS software [7]. To this end, finite length MTM cylinders and a finite length current tube, of radius $a$, current $I_{e}$, and centered at $\left(\rho_{s}, \varphi_{s}\right)$, is positioned between, and perpendicular to, 2 parallel, perfectly conducting, square plates with side length $w$ and separation $h$. Between the edges of these plates, uniform perfect matching layers of thickness $p$, and joint corners and edges, are inserted. The parameters $a=0.15 \mathrm{~mm}, h=1 \mathrm{~mm}, w=1000$ $\mathrm{mm}$, and $p=15.8 \mathrm{~mm}$. Moreover, an exact solution, see [2] for details, was obtained for the 1-cylinder configuration and included in the analysis.

\section{Results and Discussion}

It is possible to design a sub-wavelength 1-cylinder configuration capable of exciting a dipole mode resonance at a specified frequency [2]. Here, we choose such designs which excite the dipole resonances at the frequencies $f=[250,266,283,300] \mathrm{MHz}$ for cylinders $C 2, C 3, C 4$, and $C 1$, respectively. The smallest free-space wavelength is thus $1 \mathrm{~m}$. This can be accomplished if Region $i, C k$ is free space, region $o, C k$ is a SNG material with $\left(\varepsilon_{o, C k}, \mu_{o, C k}\right)=\left(\varepsilon_{0},-4 \mu_{0}\right)$, and $\rho_{i, C k}=6 \mathrm{~mm}$, for all cylinders, while $\rho_{o, C 1}=10.033 \mathrm{~mm}$, $\rho_{o, C 2}=10.024 \mathrm{~mm}, \rho_{o, C 3}=10.027 \mathrm{~mm}$, and $\rho_{o, C 4}=10.03 \mathrm{~mm}$.

The resonances of the individual cylinders are illustrated in Figure 2(a) where the radiation resistance (RR) is shown as a function of frequency for each cylinder centered at the origin and the LC, with $I_{e}=1 \mathrm{~A}$, located at $\left(\rho_{s}, \varphi_{s}\right)=\left(\rho_{o, C k}+2.5 \mathrm{~mm}, 0^{\circ}\right)$. The lossless Drude dispersion model [2] is used for the permeability $\mu_{o, C k}$ of region $o, C k$, for all $k$, such that $\mu_{o, C k}=-4 \mu_{0}$ is recovered at the respective design frequencies. The circles represent the analytical results while the full lines are the HFSS results. The agreement between the analytical and numerical methods is seen to be excellent; a similar agreement was reported in [3].
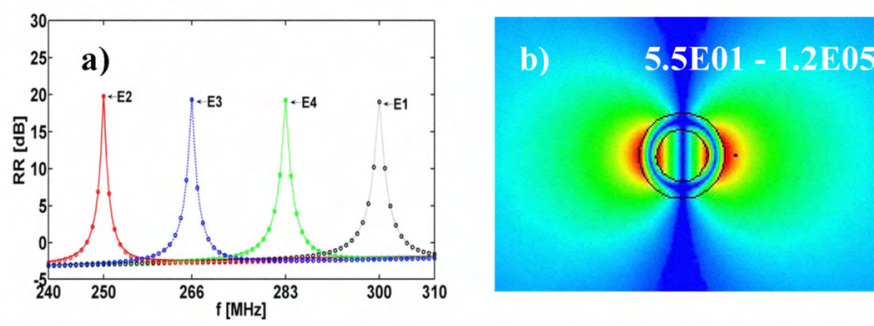

Figure 2. (a) $R R$ as a function of frequency for the resonant 1-cylinder configurations, and (b) the electric field of the resonant C2 (250 MHz) element.

It is clear that the individual 1-cylinder configurations resonate at the desired frequencies. In addition, Figure 2(b), which shows the magnitude of the electric field (the dynamic range (DR) is indicated in the figure) for the cylinder $C 2$ - which also resembles the field for other cylinders - clearly illustrates that these resonances are due to the excitation of the dipole mode in the given element.

Next, it is investigated how the individual 1-cylinder resonances from Figure 2(a) are affected when the several cylinders are combined in one configuration. 
Figure 3 shows the results for the 2-cylinder configuration made of cylinders $C 1$ and $C 2$. Specifically, Figure 3(a) and 3(b) show the RR as function of frequency for the separation distances $d=50,40,30,20,10$, and $5 \mathrm{~mm}$ with the LC located at $\left(\rho_{s}, \varphi_{s}\right)=$ $\left(\rho_{o, C k}+d / 2,0^{\circ}\right)$. For all separation distances $d$ two distinct resonances are found. For $d=50$ and $40 \mathrm{~mm}$, the resonances occur at $f=250 \mathrm{MHz}$ and $f=300 \mathrm{MHz}$ where the individual cylinders are designed to resonate. These resonances are due to the dipole mode excitation in the cylinders, as is illustrated in Figure 3 (c) and (d) where the electric field is shown for $d=40 \mathrm{~mm}$ at $f=250 \mathrm{MHz}$ (c) and at $f=300 \mathrm{MHz}$ (d) (the DR is indicated in the figure). As the separation distance $d$ decreases one and/or both resonances shift slightly away from the resonant frequencies of the individual cylinders; however, they are still due to the dipole modes in the 2 cylinders, except for $d=5 \mathrm{~mm}$ where e.g., the first resonance at $f=241 \mathrm{MHz}$ is due to a mode characterized by strong coupling between the cylinders, see Figure 3(e).
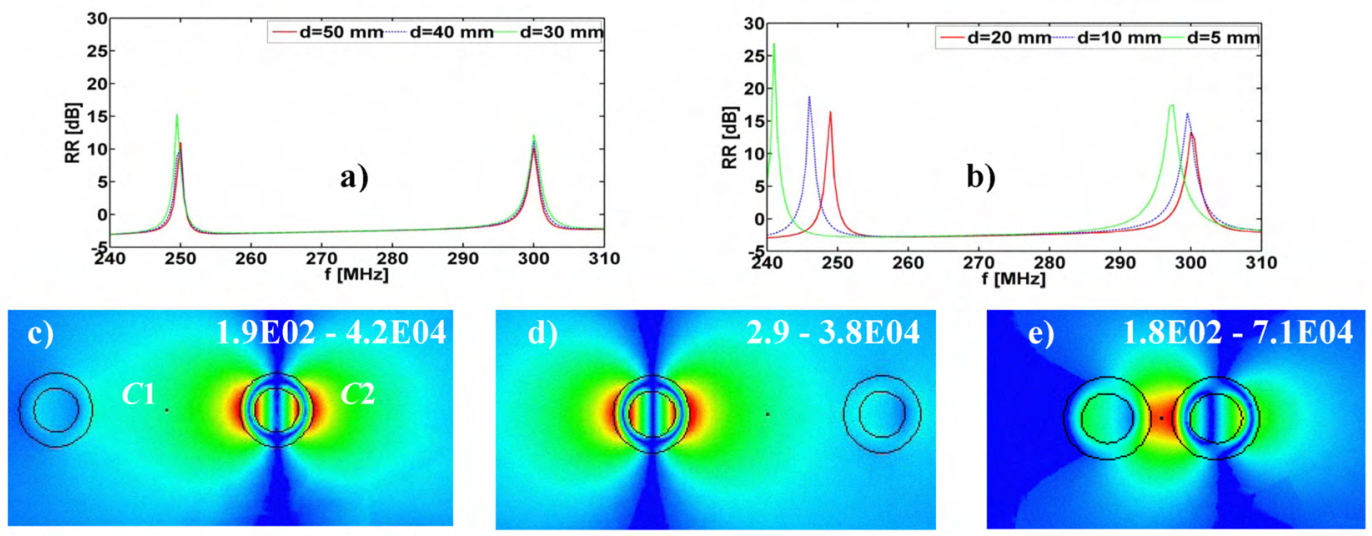

Figure 3. (a) and (b) RR as a function of frequency for the 2-cylinder configurations for different separation distances $d$. The electric field for $d=40 \mathrm{~mm}$ for $f=250 \mathrm{MHz}$ (c) and $f=300 \mathrm{MHz}$ (d) and for $d=5 \mathrm{~mm}$ and $f=241 \mathrm{MHz}$ - the latter illustrating the coupling.
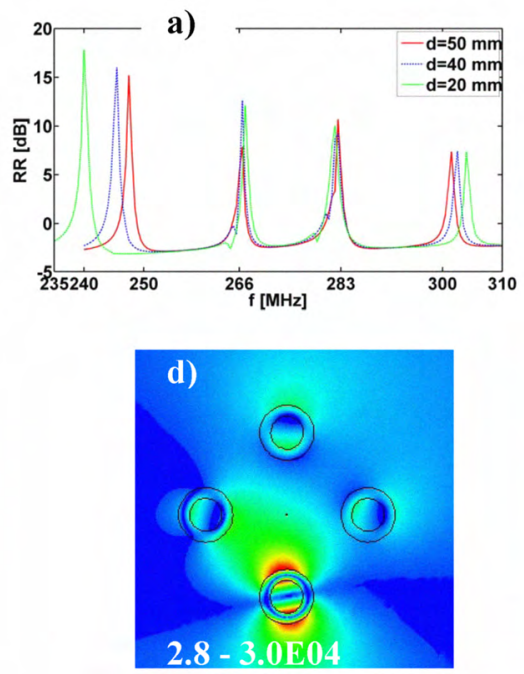
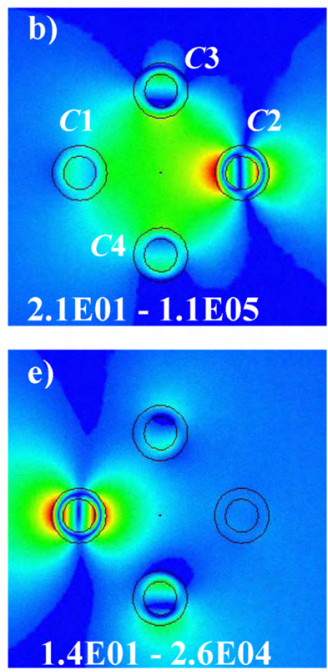
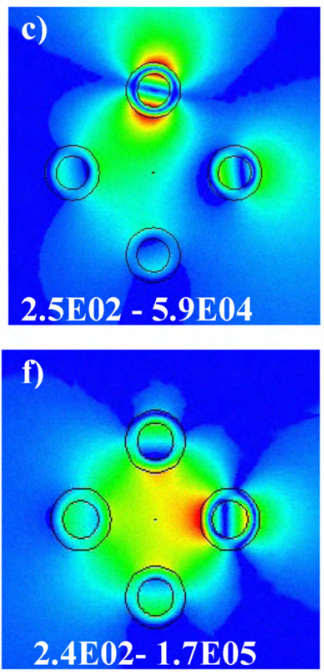

Figure 4. RR as a function of frequency (a) for the 4-cylinder configurations for different separation distances $d$. The electric field for $d=40 \mathrm{~mm}$ and $f=245.5 \mathrm{MHz}(\mathrm{b}), f=266.5$ $\mathrm{MHz}(\mathrm{c}), f=282.5 \mathrm{MHz}(\mathrm{d}), f=302.5 \mathrm{MHz}(\mathrm{e})$, and for $d=30 \mathrm{~mm}$ and $f=240 \mathrm{MHz}$ - the latter illustrating the coupling. 
Figure 4 shows the results for the 4-cylinder configuration. Specifically, Figure 4(a) shows the RR as function of frequency for $d=50,40$, and $30 \mathrm{~mm}$ when the LC is located at $\left(\rho_{s}, \varphi_{s}\right)=\left(\rho_{o, C k}+d / 2,0^{\circ}\right)$. For all separation distances $d$ four distinct resonances are found, although slightly shifted from the resonant frequencies of the individual cylinders. For a given separation $d$, this shift is larger for the 4-cylinder than for the 2-cylinder configuration. The majority of the resonances in Figure 4(a) are due to the dipole mode excitation in the individual cylinders; this is clear from Figure 4 (b)-(e), which show the electric field (the DR is indicated in the figure) for $d=40 \mathrm{~mm}$ for the frequencies at which the resonances appear $(f=245.5,266.5,282.5$, and 302.5 $\mathrm{MHz}$ ). However, for $d=20 \mathrm{~mm}$, the first resonance at $f=240 \mathrm{MHz}$ is not due to a clear dipole mode in the cylinder $C 2$, but rather to a mode which is due to coupling effects between the 4 elements.

The influence of loss in the MTMs has been assessed by the lossy Drude model [2]. The results in Figure 5 for the 4-cylinder configuration with $d=40 \mathrm{~mm}$ show that the respective resonances occur at the same frequencies as in the lossless case, but the amplitudes are, as expected, reduced.

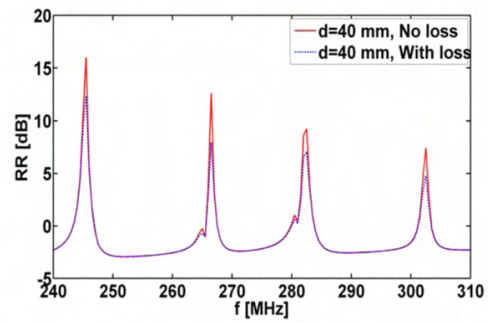

Figure 5. RR as a function of frequency for lossless and lossy 4-cylinder configuration with $d=40 \mathrm{~mm}$.

\section{Conclusions}

It has been shown that the sub-wavelength resonances of the individual MTM cylinders also occur for electrically small configurations combining 2 or 4 cylinders. For the 2and 4-cylinder configurations the overall size is $1 / 20$ and $1 / 12.5$ of the smallest wavelength, respectively. These MTM configuration thus offer the possibility for multiresonant electrically small configurations.

\section{Acknowledgments}

This work is supported by the Danish Research Council for Technology and Production Sciences within the TopAnt project.

\section{References}

[1] A. Alú, and N. Engheta, "Polarizabilities and effective parameters for collections of spherical nanoparticles formed by pairs of concentric double-negative (DNG), singlenegative (SNG) and/or double-positive (DPS) metamaterial layers," J. Appl. Phys., vol. 97, May 2005.

[2] S. Arslanagic, R. W. Ziolkowski and O. Breinbjerg, "Analytical and numerical investigation of the radiation and scattering from concentric metamaterial cylinders excited by an electric line source," Radio Sci., vol. 42, RS6S15, Nov. 2007.

[3] S. Arslanagic, and O. Breinbjerg, "Sub-wavelength resonances in polygonal metamaterial cylinders," IEEE AP-S USNC/URSI National Radio Science Meeting, San Diego, CA, USA, July 5-12, 2008.

[4] H. Wallén, H. Kettunen, and A. Sihvola, "Electrostatic resonances of negativepermittivity interfaces, spheres and wedges,"The First Intl. Congress on Advanced Electromagnetic Materials for Microwave and Optics, Rome, Italy, Oct. 22-26, 2007.

[5] A. Alú, and N. Engheta, "Cloaking and transparency for collections of particles with metamaterial and plasmonic covers," Opt. Express, vol. 15, 7578, June 2007.

[6] A. Shooshtari and A. R. Sebak "Electromagnetic scattering by parallel metamaterial cylinders,"Progress in Electromagnetic Research, PIER 57, 2006.

[7] ANSOFT HFSS, Version 10.1.3, Copyright (C) 1984-2006 Ansoft Corporation. 